\title{
Real-Time Imaging with Frequency Scanning Array Antenna for Industrial Inspection Applications at W band
}

\author{
Belen Larumbe · Jaime Laviada • Asier \\ Ibáñez-Loinaz • Jorge Teniente
}

Received: date / Accepted: date

\begin{abstract}
A real-time imaging system based on a frequency scanning antenna for conveyor belt setups is presented in this paper. The frequency scanning antenna together with an inexpensive parabolic reflector operates at the W-band enabling the detection of details with dimensions in the order of 2 millimeters. In addition, a low level of side lobes is achieved by optimizing unequal dividers to window the power distribution for side-lobe reduction. Furthermore, the quality of the images is enhanced by the radiation pattern properties. The performance of the system is validated by showing simulation as well as experimental results obtained in real-time, proving the feasibility of these kinds of frequency scanning antennas for cost-effective imaging applications.
\end{abstract}

Keywords beam steering $\cdot$ frequency scanning $\cdot$ horn antenna $\cdot \mathrm{mm}$-wave imaging W band

\section{Introduction}

The millimeter- and submillimeter-wave bands, which are part of the so-called $\mathrm{THz}$ gap, are slowly becoming more technologically relevant due to the increasing number

This work was supported by the Spanish Ministry of Economy and Competitiveness Project Nos. TEC2016-76997-C3-1-R, TEC 2013-47753-C3-1-R, RTC-2014-2110-2, and postdoctoral fellowship FPDI-2013-16278.

Belen Larumbe

IEE Department. Public University of Navarra, 31006, Pamplona (Navarra), Spain E-mail: belen.larumbe@unavarra.es

Jaime Laviada

Dept. Ingeniería Eléctrica, Universidad de Oviedo, 33203, Gijón, Spain E-mail: jlaviada@tsc.uniovi.es

Asier Ibanez-Loinaz

$\mathrm{THz}$ Technologies Research Department of Anteral, Edificio I+D Jerónimo de Ayanz, 31006, Pamplona, Spain E-mail: aibanez@anteral.com

Jorge Teniente

IEE Department. Public University of Navarra, 31006, Pamplona (Navarra), Spain E-mail: jorge.teniente@unavarra.es

Institute of Smart Cities. Public University of Navarra, Pamplona (Navarra), Spain

This is a post-peer-review, pre-copyedit version of an article published in Journal of Infrared, Millimeter, and Terahertz Waves. The final authenticated version is available online at: https://doi.org/10.1007/s10762-017-0434-6 
of applications. These frequency bands are very appealing due to multiple features including the capability of imaging through dielectric materials (paper, ceramics, wood, clothes, etc.), while it keeps millimeter or even submillimeter accuracy. Thus, $\mathrm{THz}$ imagers have quickly established on applications like concealed weapon detection on security in environments such as airports or other critical infrastructures [1].

In addition, there are a large variety of sectors such as medicine [2], viticulture [3] or passive tomography imaging [4] that could benefit from a widespread availability of $\mathrm{THz}$ imaging systems. Among these sectors, the industrial inspection in production lines have been identified as one of the most appealing ones due to the applicability for non destructive and innocuous industrial examination to detect foreign body on food products [5]. One of the current handicaps of $\mathrm{THz}$ imaging technology is the relatively high cost. Although in the long term future, technological advances are expected to allow cheap mass fabrication of $\mathrm{THz}$ components, in the meantime other cost effective approaches are needed to reduce the costs of $\mathrm{THz}$ imaging systems. One of these strategies would be reducing the number of receiver elements (and so the number of pixels) of the system, another strategy is to use a frequency scanning antenna.

This approach enables scanning capabilities along one dimension without the need of resorting to mechanical movement as it usually is required for systems based on reflectors and lenses [6]. In contrast to conventional approaches, which also enable electronic scanning, such as phased arrays [7], frequency scanning technology achieves the required phase shifts to control the beam steering by resorting to a feeding network with incremental path length [8] so that the pointing angles changes together with the frequency. Thus, the number of required components (e.g., phase shifters, mixers) is considerably reduced and so the overall cost.

Although the concept of frequency scanning antennas has been known for years $[8$, 9], the interest for $\mathrm{THz}$ imaging have hotivated a growing research in the past years, including multiple topologies $[10,11]$. It is interesting to note that although the spot is changing for each frequency and, therefore, there is not a given bandwidth for a certain position, it is possible to achieve range resolution as the expenses of cross-range resolution as well as related postprocessing algerithms as detailed in [12].

-In this framework, the authors have recently proposed a novel setup based on a frequency scanning horn antenna array together with a dish reflector [13]. In this proof of concept paper, a simple experimental setup based on a commercial TV satellite dish reflector was used for validation purposes. Two dimensional imaging was achieved by including a conventional 1D positioner but the quality of such images was quite low. -In that design, a frequency scanning antenna, formed by an array of horn antennas was considered. The array was fed by a network, which was in charge of creating the required phase shift at each frequency to achieve beam steering. The beam of the frequency scanning antenna was pointed towards an parabolic reflector, which translated the spherical wave front in the far-field of the antenna into a plane wave front. Consequently, a flat fingerprint was achieved in the observation plane.

One of the conclusions of our previous work [13] was that apart from the possibility of using a frequency scanning array for mm-wave imaging, the high level of the side lobes presented a limitation in the resultant quality that could be achieved by the image acquisition system.

In order to improve the quality, a new frequency scanning array with a fully redesigned feeding network to obtain lower side lobe levels, has been developed, analyzed and tested. Also, instead of using a commercial satellite TV reflector like in the previous work, a new reflector system has been designed, specifically for improving the 
image resolution in a conveyor belt application, and implemented using a modified commercial optical reflector. A supporting structure and a conveyor belt simulater demonstrator have been developed too, to validate the feasibility of using the proposed concept in industrial inspection applications.

In addition, the presented imaging solution has been redesigned, with a central frequency of $79 \mathrm{GHz}$ to take advantage of standard commercial components (e.g., oscillators, amplifiers, mixers, switches, etc.) to implement inexpensive front-ends so that the overall cost of the imager is greatly reduced. However, since the focus of this work is the optimized antenna and the associated system comprising the reflector, conveyor belt and real-time postprocessing, a vector network analyzer has been used to test the system.

A common problem to imaging systems based on electromagnetic waves is the blurring introduced by the radiation pattern. This problem is one of the handicaps of imaging by frequency scanning antennas due to the difficulties of achieving an a stable beam steering together with low secondary lobe level and narrow beamwidth. In passive millimeter-wave imaging, deconvolution techniques are frequently used to enhance the inherently low-resolution raw images (e.g., $[14,15]$ ) in a similar fashion to the processing of conventional optical images. In this paper, a similar approach is applied by including a deconvolution based on the measurement of the point spread function to partially cancel the blurring. To the best knowledge of the authors,this kind of enhancement techniques have not been explored in the past for imagers based on frequency scanning antennas.

The main contribution of this paper is a frequency scanning antenna combined with a conveyor belt setup, capable of acquiring a $2 \mathrm{D}$ image in W-band, process the raw data, and display it to the user, in real time, proving the feasibility of using singlemodule frequency scanning arrays for conveyor belt applications. Special attention has been paid to reduce the side lobe level by windowing the field at the aperture and to mitigate the radiation pattern distortion by postprocessing techniques.

\section{Frequency scanning system}

In order to achieve an affordable real-time imager, an evolution of our previous work [13] has been considered. The system comprises the following elements:

1. Optimized feeding network to provide a tapered field distribution.

2. Eight radiating horn antennas.

3. A parabolic reflector to provide linear scanning capabilities and enhanced directivity.

These components are described in the following sections as well as the simulation and measurement results.

\subsection{Feeding network for low sidelobe level reduction}

The frequency scanning array antenna proposed in [13] was designed to present the same amplitude in all the output ports of the distribution network of the array and, as a result of this, the theoretical sidelobe level was $13 \mathrm{~dB}$ below the main lobe level for the central frequency (Fourier transform of a rectangular distribution). The real 
Fig. 1: Example of a resultant radiation pattern from a rectangular distribution with and without Hanning windowing.

Table 1: Simulated relative amplitudes for each port employing a Hanning window for the eight output ports feeding network.

\begin{tabular}{|c|c|c|c|c|c|c|c|c|}
\hline Port number & $\# 1$ & $\# 2$ & $\# 3$ & $\# 4$ & $\# 5$ & $\# 6$ & $\# 7$ & $\# 8$ \\
\hline \hline Hanning window & 0.12 & 0.42 & 0.77 & 1 & 1 & 0.77 & 0.42 & 0.12 \\
\hline Field distribution & 0.14 & 0.48 & 0.78 & 0.99 & 1 & 0.81 & 0.53 & 0.15 \\
\hline
\end{tabular}

sidelobe level was even worse in the extreme frequencies, due to the beam scanning effect, that decreases the main lobe level as a result of misalignment between main lobe of the array factor and main lobe of the radiation pattern of the unitary elements which form the array.

In order to obtain lower side lobe levels, the most common solution relies in windowing the amplitudes of the array elements, as it can be seen in [16-19]. Several windows for the array elements amplitude distribution have been taken into consideration, e.g. Blackman, Triangular and Gaussian windows, and finally the Hanning window has been chosen as it provides a fair balance between the sidelobe level and the main lobe beamwidth (see Fig. 1).

Once the Hanning window output field aperture distribution has been chosen, an improved feeding network structure has been designed using asymmetrical power divider elements [16-19] in order to obtain the desired amplitude distribution at the output ports of the network. In Table 1, the amplitude necessary at each one of the eight output ports is shown according to Hanning distribution. Asymmetrical T-junctions have been developed varying the width and the length of the junction output branches. A particular asymmetrical power divider configuration has been chosen thinking on easiness of fabrication with standard milling processes and good performance. 


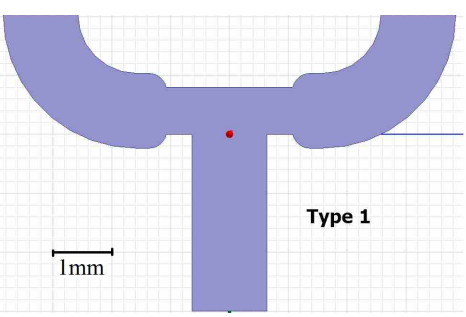

(a)

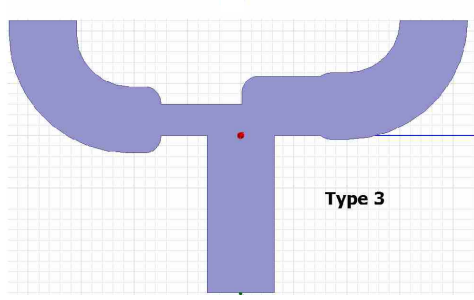

(c)

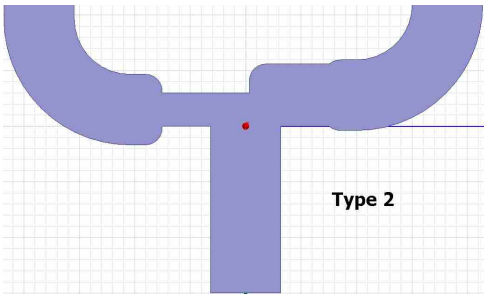

(b)

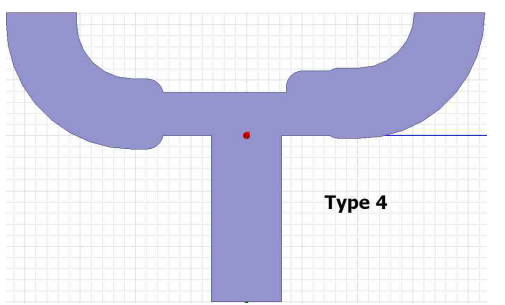

(d)

Fig. 2: Power dividers element divider elements used to achieve the desired amplitude window at the output ports of the distribution network.

Table 2: S-parameters simulation for each power divider at $79 \mathrm{GHz}$.

\begin{tabular}{|c|c|c|c|c|}
\hline & Type \#1 & Type \#2 & Type \#3 & Type \#4 \\
\hline \hline S21 & $-3 \mathrm{~dB}$ & $-6.29 \mathrm{~dB}$ & $-6.45 \mathrm{~dB}$ & $-3.6 \mathrm{~dB}$ \\
\hline S31 & $-3 \mathrm{~dB}$ & $-1.17 \mathrm{~dB}$ & $-1.14 \mathrm{~dB}$ & $-2.48 \mathrm{~dB}$ \\
\hline
\end{tabular}

Fig.Figure 2 shows the four different junctions used. Due to the symmetry of the windowed amplitude distribution (see Table 1), only 4 different junctions are necessary to obtain the desired power levels at the output ports. The resultant S-parameters for this T-junctions are shown in Fig. 3 and Table 2.

In Fig. 4, the layout of the complete system is presented. The feeding network is composed of sections of WR-10 waveguides $(2.54 \mathrm{~mm} \times 1.27 \mathrm{~mm})$. The power dividers have been designed to achieve the desired output amplitude in each one of the output ports. It is worth noting that the conductor losses, assuming an aluminum structure, yield a difference of $0.63 \mathrm{~dB}$ between the shortest and the longest path. Since this difference is small, it has been neglected and, consequently, the power balance has been designed based only on the asymmetric power dividers, without compensating the effect of that loss.

The results for the simulated amplitude for the eight output ports of the feeding network including losses are shown in Table 1, the slight imbalance can be seen. The length of the paths of the feeding network has been chosen to achieve a frequency scanning angle of 50 degrees in a band from 77 to $81 \mathrm{GHz}$. 


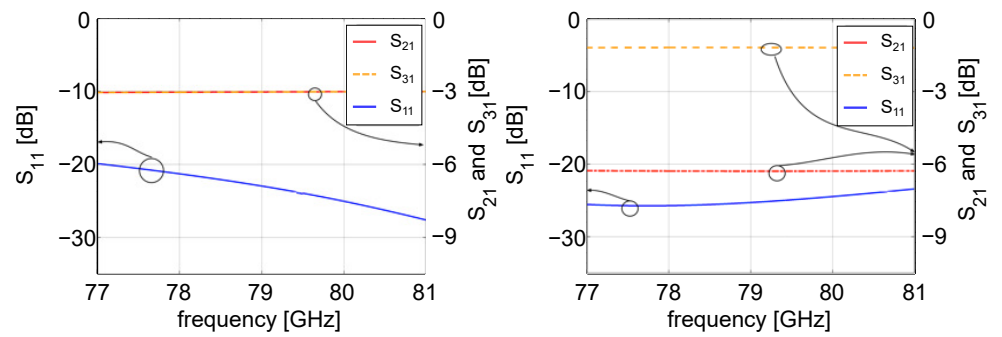

(a)

(b)

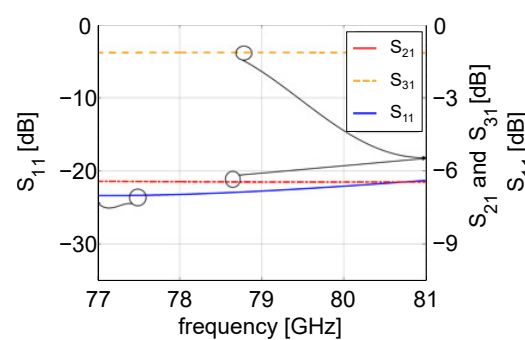

(c)

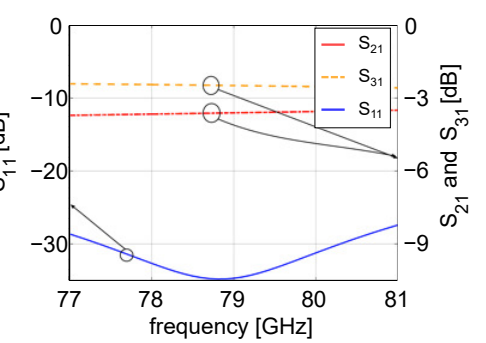

(d)

Fig. 3: S-parameters simulation for the four power dividers: a) Type 1; b) Type 2; c) Type 3 and d) Type 4 .

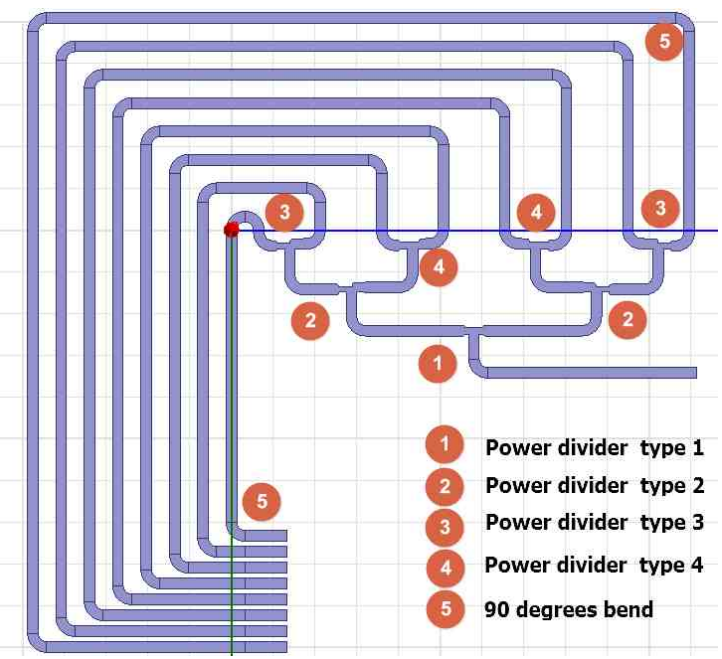

Fig. 4: Complete layout of the feeding network for the 8 WR10 apertures. 


\subsection{Radiating elements}

Regarding the eight horn elements, a W-band scaled version of the eight H-plane horns described in [13] has been employed since the imaging performance has a minor dependence on this component that can be easily fabricated by means of electro-discharge machining (EDM). This antenna array has been designed to produce a narrow and symmetric radiation pattern when all the eight ports present the same amplitude and phase. The input port of each individual horn is $2.54 \mathrm{~mm} \times 1.27 \mathrm{~mm}$ (WR-10 waveguide) and the output aperture is $27.5 \mathrm{~mm} \times 15 \mathrm{~mm}(27.5 \mathrm{~mm} \times 1.7 \mathrm{~mm}$ for each one of the pyramidal horns, so nearly an H-plane horn antenna array).

\subsection{Parabolic reflector}

As the aim of this work is to design a cost-effective imaging system, the use of a commercially available parabolic reflector was desirable. According to the desired resolution level and the size of the horn array, it was convenient to choose a reflector with relatively high $f / D$ ratio and diameter, $D$. However, low cost commercially available reflectors with large $f / D$ ratio and good surface reflectance characteristics to not interfere with this high frequency employed, had often a small diameter, not large enough for this application. Finally, an Edmund Optics parabolic reflector with $f / D=0.25$ and diameter $D=609.6 \mathrm{~mm}$ has been found to provide a fair trade-off between both parameters.

Several simulations have been performed with the Ticra's GRASP software [20] in order to determine the optimal reflector configuration. Finally, an offset configuration has been chosen, determining that the 50 degrees of the total frequency scan is translated into a linear sweep of approximately $20 \mathrm{~cm}$ in the image plane placed at $0.5 \mathrm{~m}$ from the reflector.

Then, the Edmund Optics symmetrical parabolic reflector has been cut to obtain an offset reflector configuration, as can be seen in Fig. 5 .

\subsection{Simulation Results}

The 8-horn pyramidal array with the feeding network has been simulated and optimized using Ansys HFSS [21]. In Figs. 6 and 7, the H-plane and E-plane radiation patterns from 77 to $81 \mathrm{GHz}$ are shown. It can be seen that both in the E- and H-planes, the beamwidth is very similar at each frequency. In the E-plane, the frequency scanning can be observed as well as the impact of the Hanning windowing in the sidelobe level which is $1917 \mathrm{~dB}$ below the main beam value in the worst case and, therefore, a significant improvement has been obtained compared with respect to the $8 \mathrm{~dB}$ simulated value presented in [13].

\subsection{Prototype Fabrication}

The antenna has been fabricated in aluminum, using milling for the two E-plane split halves of the feeding network, and wire EDM machining for the single piece horn array. The system is completed with the reflector, as well as metallic profiles and $3 \mathrm{D}$ printed 


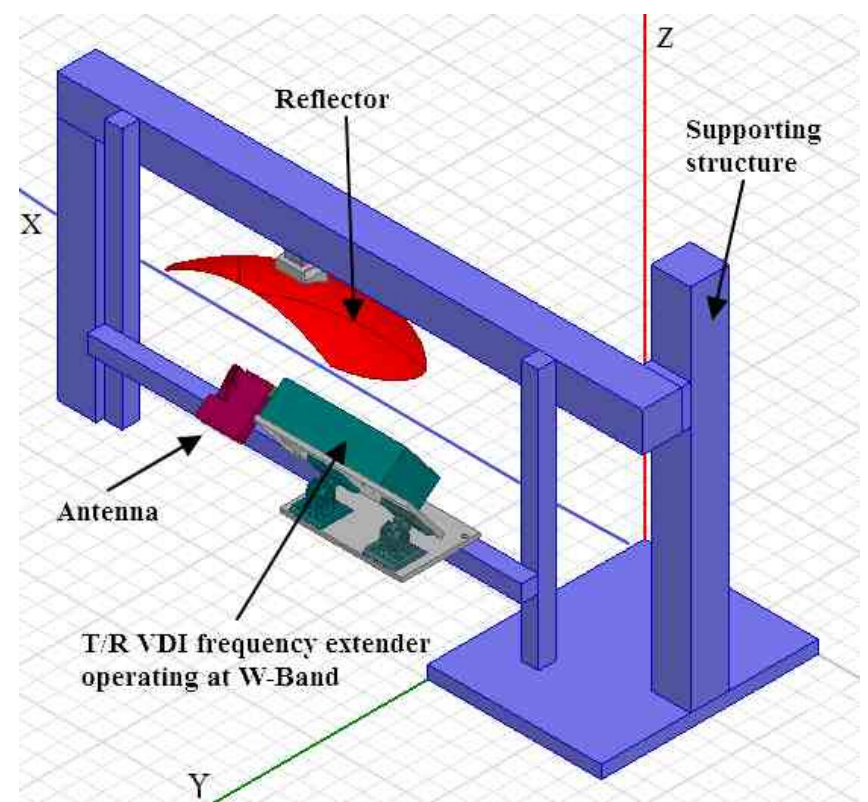

Fig. 5: Complete imaging system including the antenna and the reflector, no movable parts are needed in the RF chain, the image is formed by frequency scanning in the $\mathrm{X}$ axis and the conveyor belt movement in the $\mathrm{Y}$ axis.

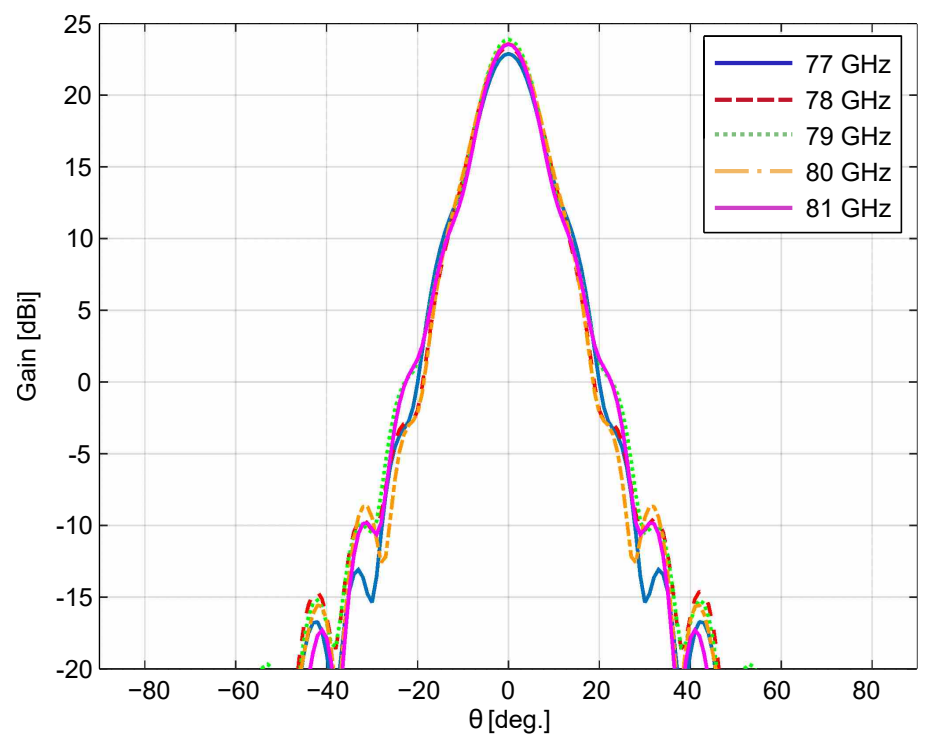

Fig. 6: Simulated H-plane radiation pattern. 


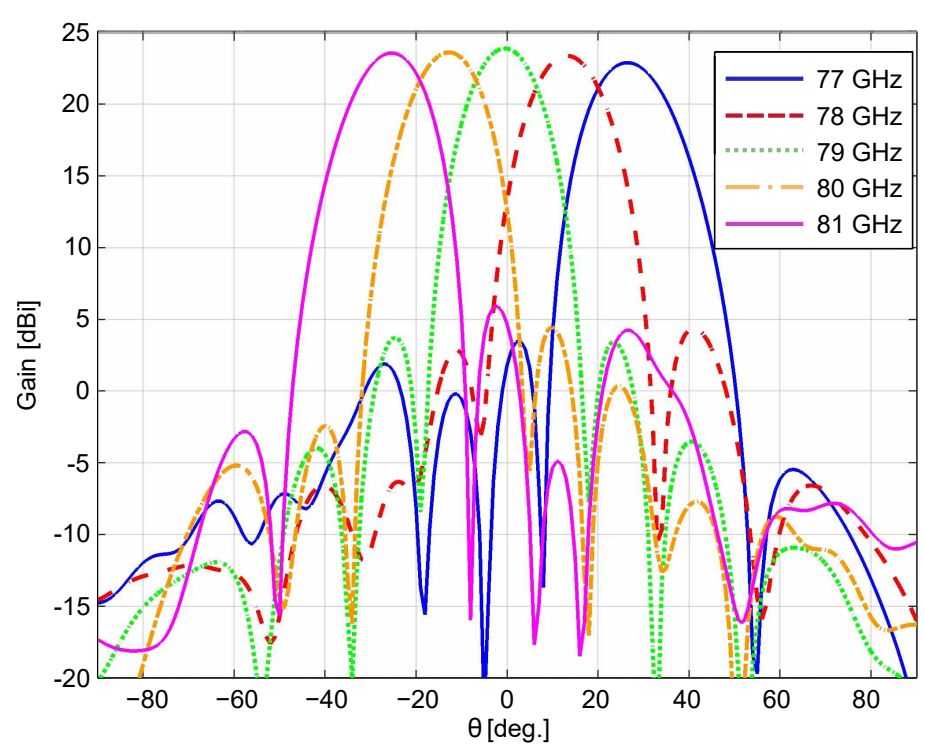

Fig. 7: Simulated E-plane radiation pattern for a $4 \mathrm{GHz}$ beamwidth.

components for structural support and attachment. A conveyor belt is placed under the structure to move the targets through the scanning area. Fig. 8 shows a picture of the complete system.

\subsection{Measurements}

The horn array antenna and the complete system have been measured in a planar near-field test setup, in order to verify agreement with the simulation results, and also to properly characterize the system for the image processing that will be done in the next section.

The measurement has been performed with an Agilent PNA-X N5242A network analyzer equipped with two VDI (Virginia Diodes) millimeter wave frequency extenders operating at the $\mathrm{W}$ band.

Fig. 9 shows the measured E-plane radiation pattern of the horn array and the feeding network. A frequency shift of about $1 \mathrm{GHz}$ can be observed due to the thigh manufacturing tolerances required by the incremental path length strategy of the feeding network. Also, measured losses are around $2 \mathrm{~dB}$, when compared to the simulations. The measured sidelobe level is higher than in the simulations due to imbalances in the fabricated power dividers, but is below more than $12 \mathrm{~dB}$ compared to the main beam in the worst case, providing an important improvement compared to [13] where the measured worst case sidelobe level was only $5 \mathrm{~dB}$ below the main beam. Figures 10 and 11 show the measured beam spots at the image plane of the system that will be used for imaging results postprocessing. This radiation patterns, in contrast to the horns radiation patterns, are only reported in the near-field. In particular, they are only reported at the conveyor belt plane as it is the area to be monitored. 


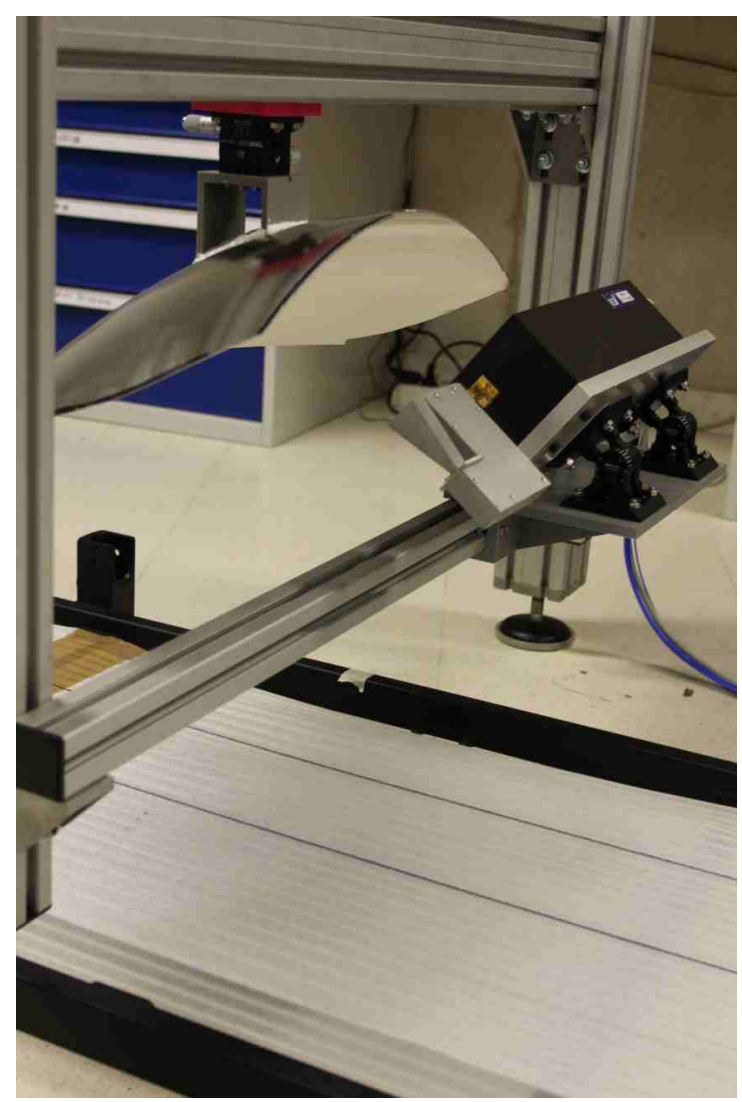

Fig. 8: Complete system with the conveyor belt at the bottom.

\subsection{Cost considerations}

When comparing the proposed system with a traditional multipixel array we can take Fig. 11 as a reference to determine that at least 9 separate pixels would be required.

Although the cost and complexity of a frequency tunable transceiver is higher than in the case of a fixed band one, the obtained pixel reduction ratio is of 9 to 1 , decreasing the overall cost of the system, when using the frequency scanning approach.

Moreover, it is expected that the use of MMIC or flip-chip W-band automotive radar commercial components could drive down the cost of every tunable $\mathrm{W}$ band $\mathrm{T} / \mathrm{R}$ module close to the cost of its fixed frequency counterpart, making the proposed approach even more attractive from a production cost point of view.

\section{Image postprocessing}

Although the radiation pattern of the presented frequency scanning antenna exhibits a significant improvement with respect to [13], the spot size is still relatively large and, consequently, it will result in a distortion of theimages. In this section, it is shown 


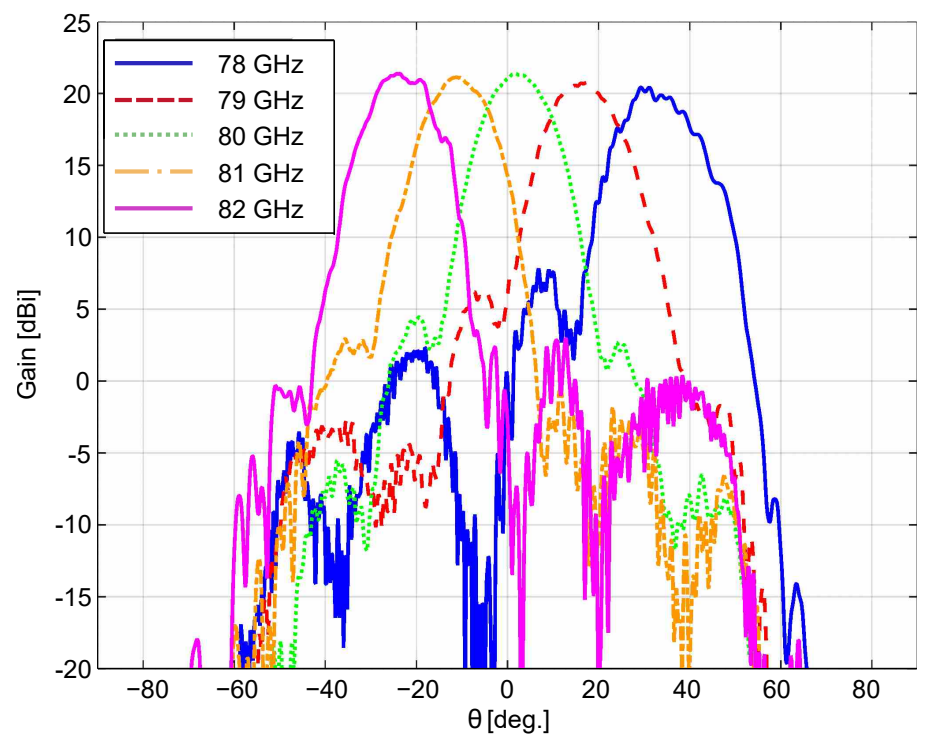

Fig. 9: Measured E-plane radiation pattern of the horn array.

how the quality of images can be improved by applying an appropriate deconvolution. This postprocessing, requires a number of steps and approximations, to conveniently produce a two-dimensional image from the acquisition of the $S_{11}$ parameter for multiple frequencies at a given time rate.

\subsection{Radiation pattern deconvolution}

The amplitude received by the frequency scanning antenna working as a monostatic radar is given by:

$$
M(f, \Delta y)=\int_{S}\left|E\left(x, y, z_{0}\right)\right|^{2} \Gamma_{\mathrm{obj}}(x, y+\Delta y) d s,
$$

where $\Gamma_{\mathrm{obj}}$ is the reflectivity of the object, $\Delta y$ is the displacement of the object due to the movement of the conveyor belt and $E$ is the electric field at the plane of the object under test $z=z_{0}$. The square superscript is included in (1) due to the monostatic setup.

Since the field $E$ can be measured in a calibration stage and $M$ is the (known) measured signal, eq. (1) depicts a standard inverse scattering problem modeled by an integral equation.

The solution of this kind of problems is usually based on discretizing the unknown variable to yield a matrix equation [22]. Nevertheless, this approach is challenging as it entails dealing with poor conditioned systems of equations [22]. Consequently, this technique is mainly useful for systems providing high signal to noise ratios and enhanced by an appropriate regularization scheme. Furthermore, the solution of these matrix problems is usually computationally expensive and, therefore, they are not convenient for a real-time imager. 


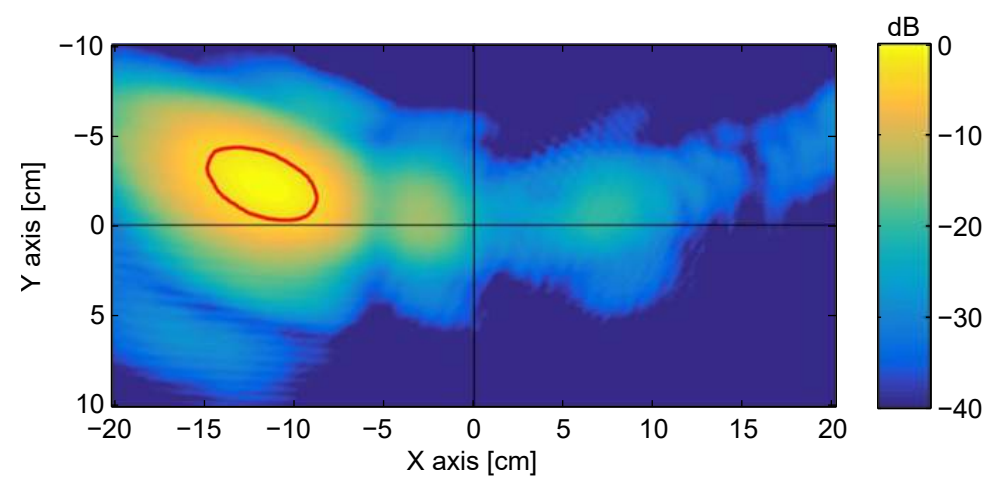

(a)

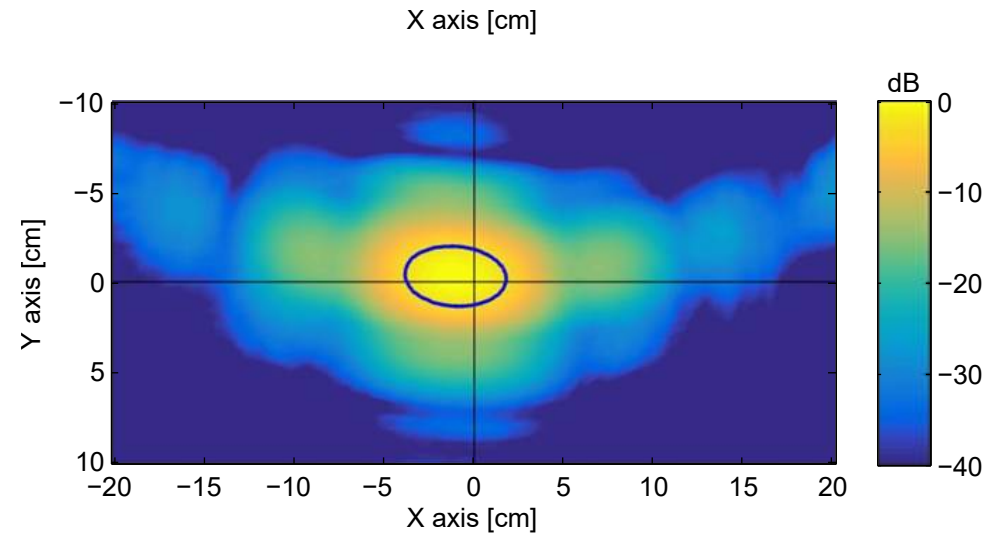

(b)

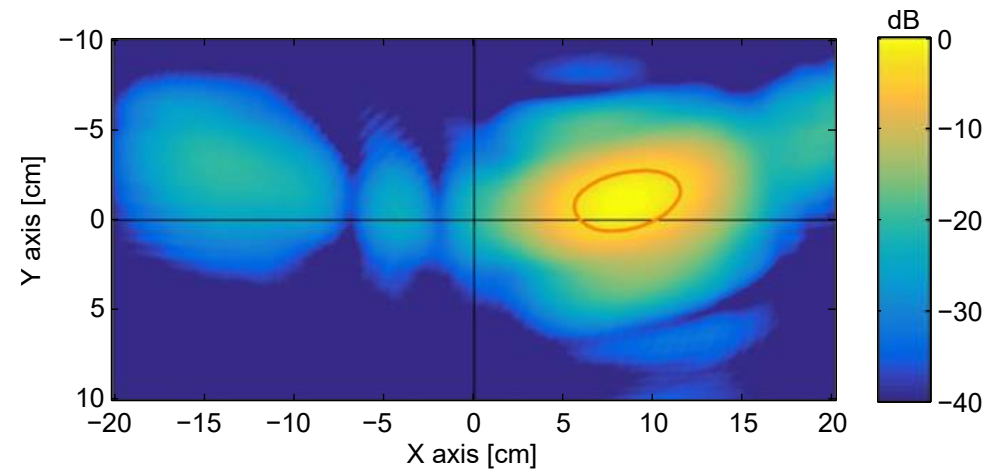

(c)

Fig. 10: Measured near-field radiation pattern in the image plane after the mirror at a) $78 \mathrm{GHz}$ b) $80 \mathrm{GHz}$ c) $82 \mathrm{GHz}$. 


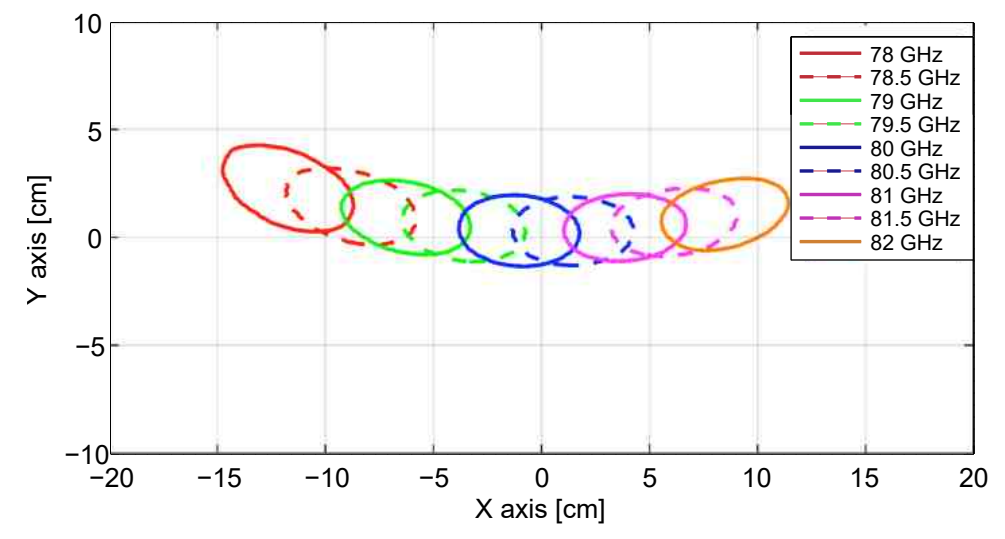

Fig. 11: Measured near-field beamspots (-3 dB contour plot) in the image plane.

In order to achieve a robust and efficient solution of (1), some simplifications will be firstly performed. In particular, it is assumed that the spot moves linearly along the $x$-axis.

Thus, the position of the spot can be described as a function of the frequency as:

$$
\mathbf{r}_{\text {spot }}=a\left(f-f_{c}\right) \hat{x},
$$

wherein $a$ accounts for the spot movement rate and $f_{c}$ is the center frequency. This simplification is supported by Fig. 12 depicting the position of the maximum of the spot for an acquisition at $z=50 \mathrm{~cm}$. It is clearly seen that the position along the $x$-axis fits well a linear dependency. Applying a linear regression to the previous curve, it has been obtained that $a=4.93 \mathrm{~cm} / \mathrm{GHz}$. In addition, the position of the spot along the $y$-axis suffers only a moderate movement.

Next, it is also assumed that the spot shape does not depend on the frequency. Although the spot suffers some tilt when it moves along the XY plane (see Fig. 11), this effect is small along the center frequency as the curve of the spot maximum is almost flat. Under the previous simplification, eq. 1 can be expressed as:

$$
M(\Delta x, \Delta y) \approx \int_{x} \int_{y} P S F\left(x+\Delta x, y, z_{0}\right) \Gamma_{\mathrm{obj}}(x, y+\Delta y) d x d y,
$$

where the displacement along $x$ is related to the frequency by $\Delta x=a\left(f-f_{c}\right)$ and $P S F$ is a point spread function (PSF) that is the responsible of the blurring of the image. This function is related to field of the antenna by:

$$
P S F=\left|E\left(x, y, z_{0}, f_{c}\right)\right|^{2} .
$$

In order to achieve a flexible implementation, it is convenient to approximate the PSF by an analytic function so that the values can be easily tuned in the final implementation. After observing the electric field shown in Fig. 9, it has been observed that a Gaussian function should provide a good fitting. As shown in the past, imagers affected by this kind of blur are good candidates to be enhanced by deconvolution [15]. Hence, the expression for the point spread function is approximated by:

$$
P S F(x, y) \approx \frac{1}{2 \pi \sigma_{x} \sigma_{y}} \exp \left(-\frac{x^{2}}{2 \sigma_{x}^{2}}-\frac{y^{2}}{2 \sigma_{y}^{2}}\right),
$$




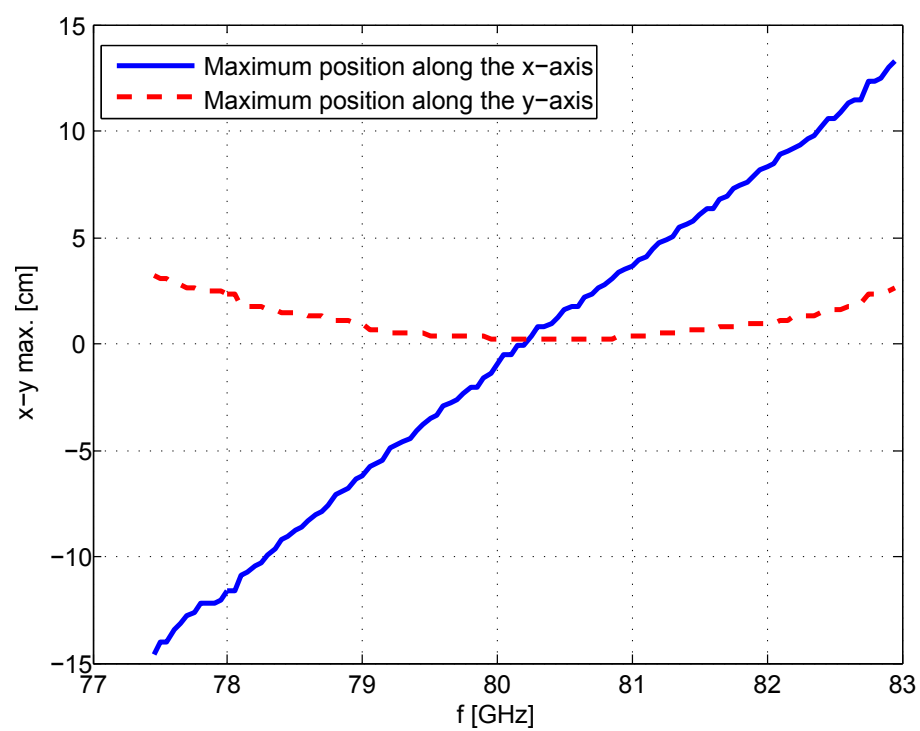

Fig. 12: Position of the maximum of the spot in the $x$ - and $y$-axes versus frequency at $z=50 \mathrm{~cm}$.

where the values of $\sigma_{x}=2.3 \mathrm{~cm}$ and $\sigma_{x}=1.34 \mathrm{~cm}$ are found to provide an accurate model as shown in Fig. 13.

Taking into account the symmetry of the point spread function, the integral equation described by (3) can be expressed as a convolution:

$$
M(x, y) \approx P S F(x, y) * \Gamma_{\mathrm{obj}}(x, y)
$$

Thus, the solution of the integral equation described by (1) can be approximately solved by deconvoluting the measured signal with a known function. This kind of problem is well known in a number of fields including image processing or astronomy and, therefore, a large number of robust and efficient algorithms is available.

It is important to remark that these algorithms can partially remove the effect of the blurring introduced by the PSF. Nevertheless, the real image (or, in this case, the object reflectivity) is not completely recovered as the PSF performs a lowpass filtering and, consequently, high frequencies cannot be retrieved.

One of the most widespread deconvolution approaches for enhancing mm-wave images is the blind deconvolution $[14,15]$ which does not require the a priori knowledge of the PSF. Nevertheless, the radiation pattern of the antenna has been characterized (see previous section) in the proposed imager. Thus, it is more convenient to resort to deconvolution approaches that can take advantage of the knowledge of the PSF. In particular, the Lucy-Richardson deconvolution [23-25] has been used in this work. This algorithm relies on an iterative refinement of the solution, which is given by the following expression:

$$
\hat{\Gamma}_{\mathrm{obj}}^{(t+1)}(x, y)=\hat{\Gamma}_{\mathrm{obj}}^{(t)}(x, y)\left(\frac{|M(x, y)|}{\hat{\Gamma}_{\mathrm{obj}}^{(t)}(x, y) *|P S F(x, y)|} *|P S F(x, y)|\right),
$$




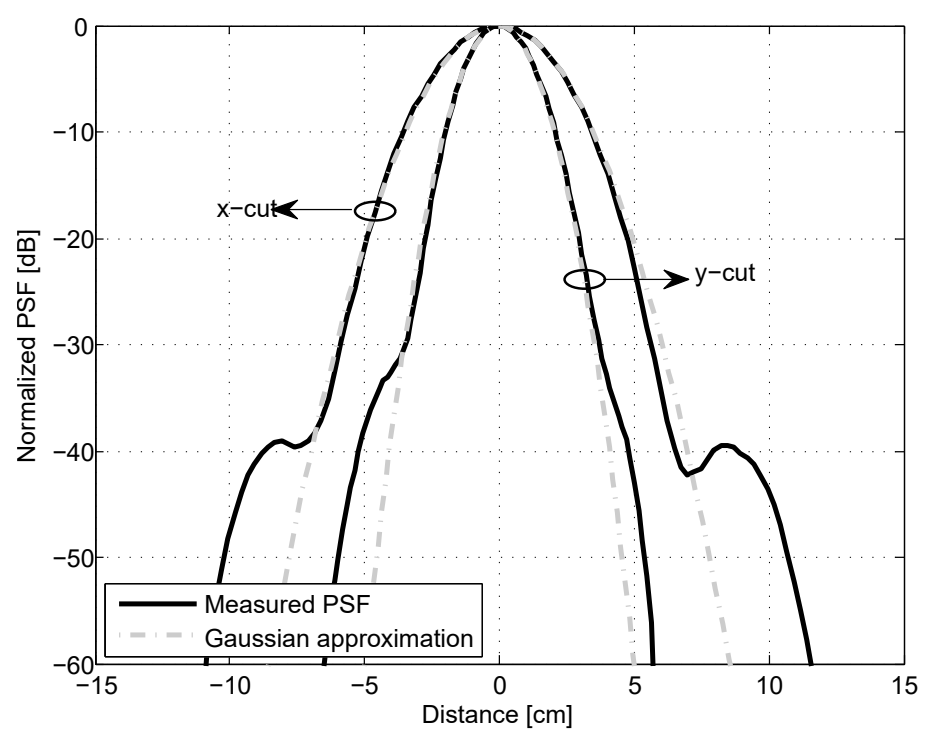

Fig. 13: Approximation of the point spread function by a Gaussian function with $\sigma_{x}=$ $2.3 \mathrm{~cm}$ and $\sigma_{y}=1.34 \mathrm{~cm}$. The point spread function has been measured at $f_{c}=$ $80.35 \mathrm{GHz}$

where the products and division are defined as element wise and $\hat{\Gamma}_{\mathrm{obj}}^{(t)}$ is the estimation of the object reflectivity at iteration $t$. Unless otherwise specified, the number of iterations is fixed to 10 in this work. The symmetry of the PSF has been used to further simplify the original expression [24,23]. Although the phase of the measured signal would provide some additional information, which could improve the quality of the solution, it has been observed that this phase is very sensitive to any instability (e.g., vibrations of the conveyor belt). For this reason, amplitude-only data is used in (7).

Finally, it is also relevant to mention that the postprocessing includes a preliminary stage where the scattered field without any object is measured and, after that, it is subtracted to any measurement. In addition, a time gating is also applied. The purpose of both techniques is to mitigate the impact of unwanted reflections happening inside or outside the antenna.

\subsection{Results for simulated data}

In order to validate the performance of the proposed deconvolution to improve the quality of the imaging, let us firstly consider a simulated example. In this case,the object is a ring with external radius of $5 \mathrm{~cm}$ and internal radius of $2 \mathrm{~cm}$. The reflectivity is set to one for all the points inside the object. The simulated data is computed by introducing the electric field acquired as described in section 2.5 and the aforementioned reflectivity into (1).

Only the data from $77.45 \mathrm{GHz}$ and to $82.95 \mathrm{GHz}$ is used yielding a total of 111 frequency points. The data is sampled along the y-axis with a spacing of $1.5 \mathrm{~mm}$. Finally, white Gaussian noise is added to simulate a signal to noise ratio of $30 \mathrm{~dB}$. 

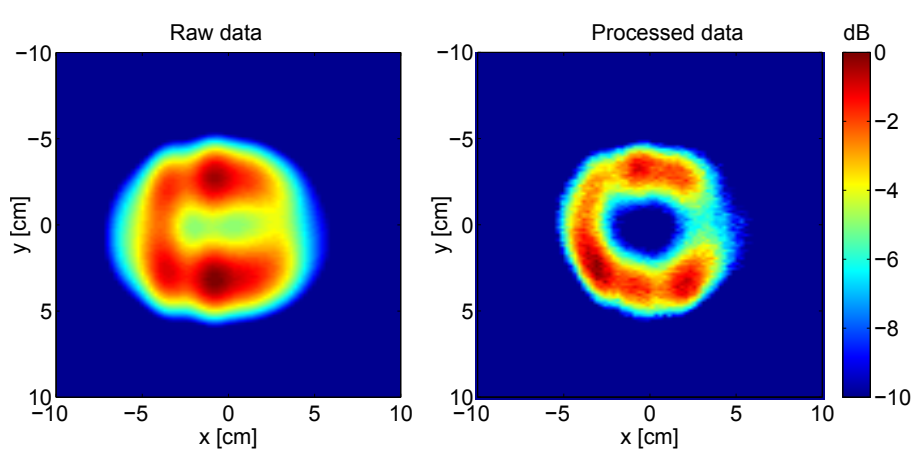

Fig. 14: Normalized images for a simulated object with ring-shape.

The simulated raw and postprocessed data are shown in Fig. 14. The raw image clearly resembles the real object but it lacks of some important details. On the other hand, the deconvoluted image reveals a clear improvement even if the real object is not perfectly retrieved due to the approximations and deconvolution limitations.

\section{Real-time image results}

In order to validate the performance of the system, the frequency scanning antenna is connected to a vector network analyzer (VNA) which is set to sample 201 frequency points ranging from $75 \mathrm{GHz}$ to $85 \mathrm{GHz}$. After subtracting the reflection coefficient measured without an object, a time gating from $7.5 \mathrm{~ns}$ to $10 \mathrm{~ns}$ is set to filter undesired echoes from the floor as well as internal reflections in the antenna. The intermediate frequency (IF) filter bandwidth is set to $4 \mathrm{kHz}$ resulting in a sweep time of $42.813 \mathrm{~ms}$.

The objects that have been measured are a stick of aluminum foil with a diameter between $7 \mathrm{~mm}$ and $10 \mathrm{~mm}$ and length of $22 \mathrm{~cm}$, a piece of glass with dimensions of approximately $7 \mathrm{~mm} \times 11 \mathrm{~mm}$, two aluminum foil balls with a diameter of approximately $3 \mathrm{~mm}$ and a hexagonal M4 nut (see Fig. 15).

The objects are placed on the conveyor belt (see Fig. 16) that is fed by DC source at $5 \mathrm{~V}$ resulting in a speed of approximately $4 \mathrm{~cm} / \mathrm{s}$. The obtained values for the modified time-gated $S_{11}$ parameter for the raw and processed data are shown in Fig. 17. In these images, it is observed that the objects can be clearly detected by the system. Although the deconvolution cannot completely retrieve the objects, it significantly improves the resolution, especially along the $x$-axis, where the PSF is wider.

\section{Conclusions}

The results presented in this paper show the feasibility of an affordable scanning array imaging for conveyor belt setups working at $79 \mathrm{GHz}$. The system comprises a frequency scanning array with optimized field distribution at the aperture to reduce the side lobe level, an inexpensive reflector and a real-time postprocessing technique providing capabilities to detect small objects $(<3 \mathrm{~mm})$ on-the-fly. 


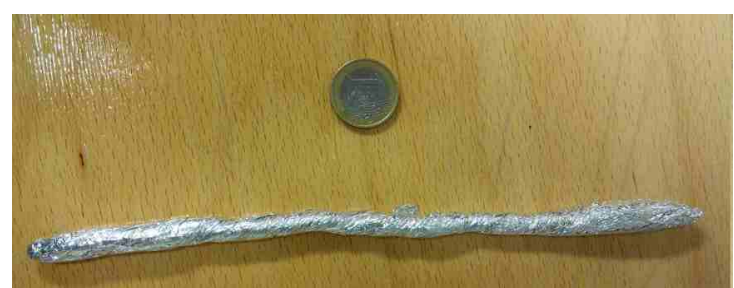

(a)

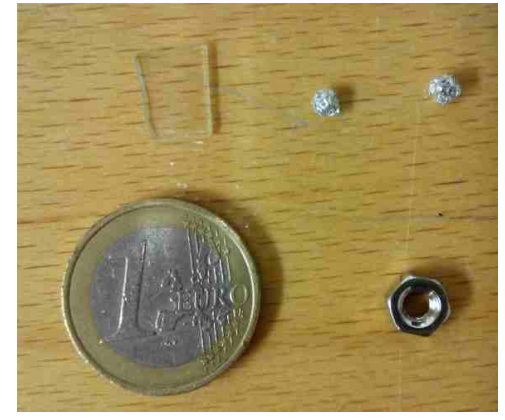

(b)

Fig. 15: Measured objects with enhanced imaging.

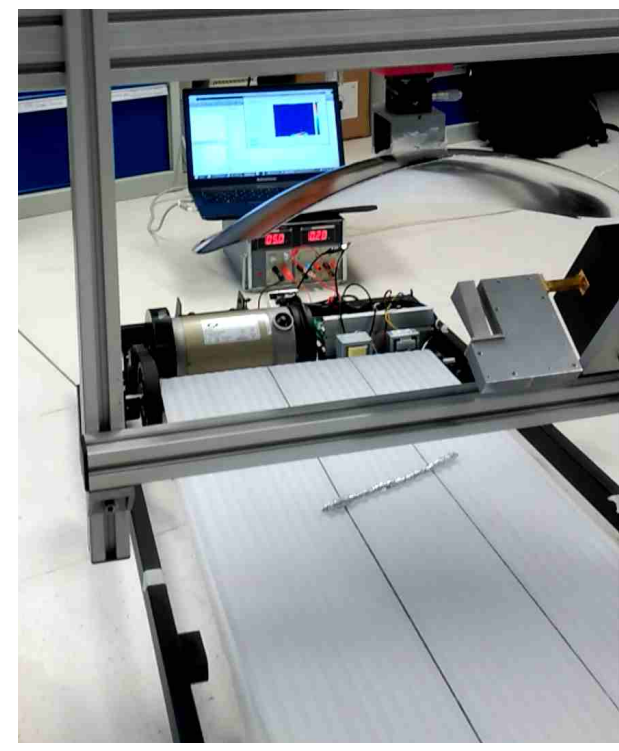

(a)

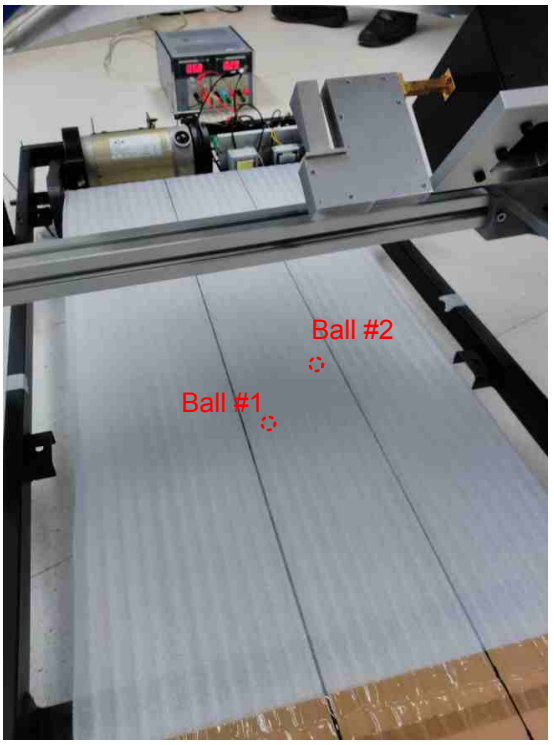

(b)

Fig. 16: Setup including objects: (a) aluminum foil stick with a tilt of approximately $45^{\circ}$; (b) aluminum foil balls with a separation of approximately $7 \mathrm{~cm}$ and $6 \mathrm{~cm}$ for the $x$ - and $y$-axes respectively . 

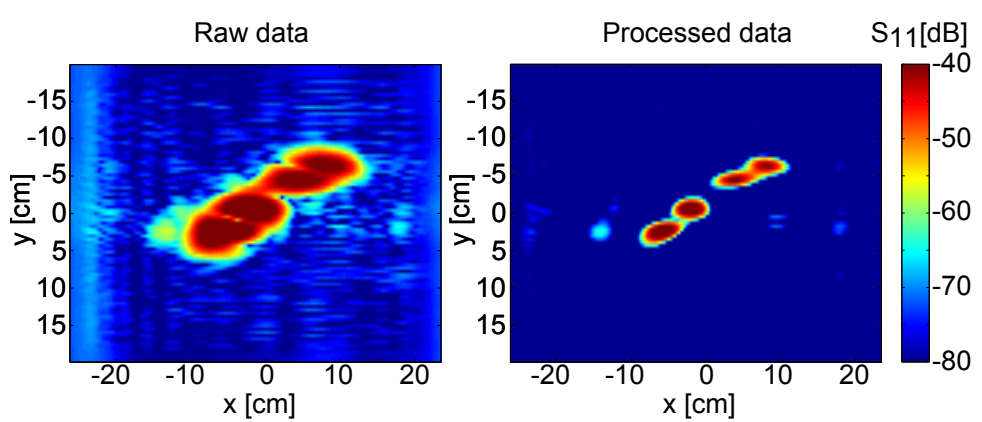

(a)
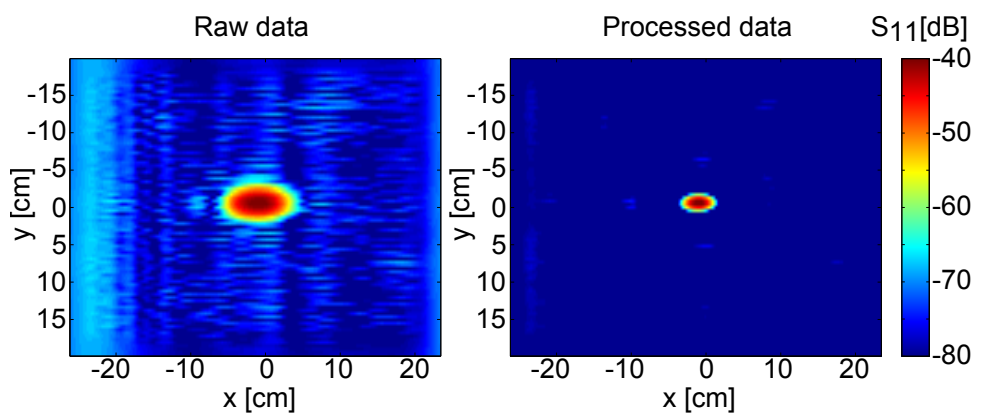

(b)
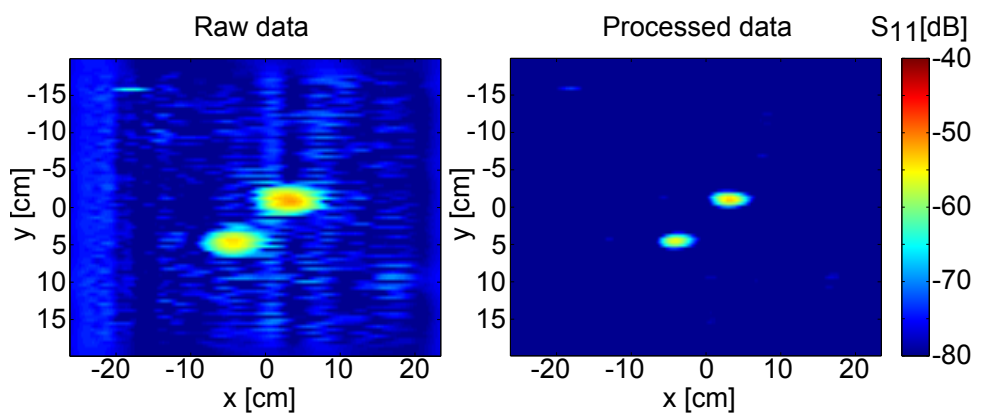

(c)
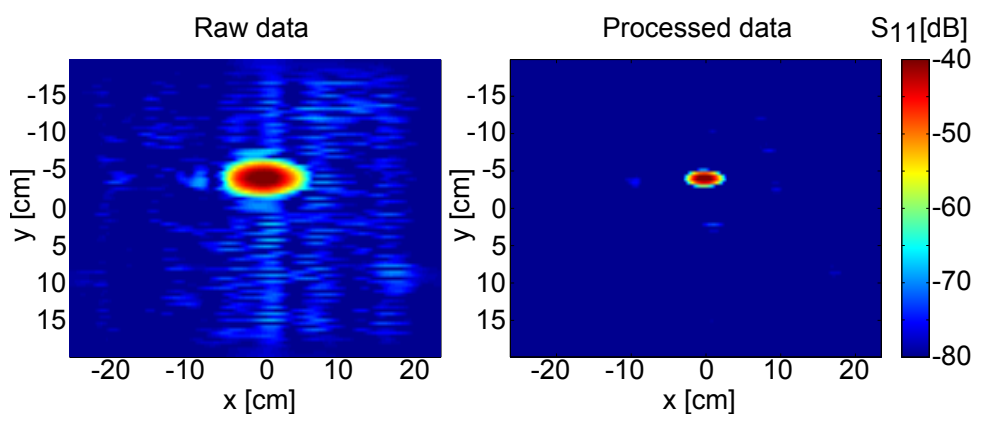

(d)

Fig. 17: Measured and processed $S_{11}$ after the subtraction of the value without object. Results are reported for: (a) aluminum foil stick; (b) piece of glass; (c) aluminum foil balls; (d) M4 nut. 
The frequency scanning approach could lead to a significant cost reduction with regards to traditional multipixel arrays, specially in applications requiring a high number of pixels.

The system has been validated by means of several targets including $2 \mathrm{~mm}$ diameter metallic balls, and $0.5 \mathrm{~mm}$ thickness glass fragments with good image quality in all the cases, giving an example of the potential industrial applications. However, there would still be margin for improving the system in terms of resolution by, for example, further optimization of the reflection system, or increasing the reflector size (at the expenses of a larger system size), or increasing the number of elements of the array (also increasing the cost and complexity of the feeding network and the system size).

\section{References}

1. K.B. Cooper, R.J. Dengler, N. Llombart, B. Thomas, G. Chattopadhyay, P.H. Siegel, IEEE Transactions on Terahertz Science and Technology 1(1), 169 (2011)

2. E. Pickwell, V.P. Wallace, J. Phys. D: Appl. Phys. 39, R301 (2006)

3. B.S. Peter, S. Yngvesson, P. Siqueira, P. Kelly, A. Khan, S. Glick, A. Karellas, IEEE Journal of Biomedical and Health Informatics 17(4), 785 (2013)

4. X. Yin, B.W.H. Ng, B. Ferguson, S.P. Mickan, D. Abbott, IEEE Sensors Journal 7(3), 342 (2007)

5. D. Etayo, J.C. Iriarte, I. Palacios, I. Maestrojuán, J. Teniente, I. Ederra, R. Gonzalo, in 2011 IEEE MTT-S International Microwave Workshop Series on Millimeter Wave Integration Technologies (2011), pp. 172-175

6. H. Tran, F. Gumbmann, J. Weinzierl, L. Schmidt, Spatial, in Proc. German Microwave Conference (Karlsruhe, 2006)

7. R.J. Mailloux, Phased Array Antenna Handbook (Artech House, 2nd ed., 2005)

8. A. Ishimaru, H.S. Tuan, IRE Transactions on Antennas and Propagation 10(2), 144 (1962)

9. K.L. Klohn, R.E. Horn, H. Jacobs, E. Freibergs, IEEE Transactions on Microwave Theory and Techniques 26(10), 764 (1978)

10. E.D. Cullens, L. Ranzani, K.J. Vanhille, E.N. Grossman, N. Ehsan, Z. Popovic, IEEE Transactions on Antennas and Propagation 60(8), 3647 (2012)

11. R. Camblor, S.V. Hoeye, C. Vázquez, G. Hotopan, M. Fernández, F. Las-Heras, Journal of electromagnetic Waves and Applications 26(4), 468 (2012)

12. Y. Álvarez et al., IEEE Transactions on Antennas and Propagation 61(11), 5689 (2013)

13. B. Larumbe-Gonzalo, A. Ibáñez-Loinaz, R. Gonzalo, J. Teniente, Microwave and Optical Technology Letters 56(12), 2851 (2014)

14. L. Spinoulas, B. Amizic, M. Vega, R. Molina, A.K. Katsaggelos, in 2012 Proceedings of the 20th European Signal Processing Conference (EUSIPCO) (Bucharest, Hungary, 2012), pp. 1414-1418

15. H. Fang, L. Yan, International Journal for Light and Electron Optics 125(3), 1454 (2014)

16. S. Gruszczynski, K. Wincza, K. Sachse, IEEE Antennas and Wireless Propagation Letters $\mathbf{5}(1), 430(2006)$

17. R.K. G. Jain, D.J. Ghosh, IOSR Journal of Electronics and Communication Engineering $\mathbf{9}(3), 57(2014)$

18. F. Zulkifli, T. Hidayat, Basari, E.T. Rahardjo, in Proc. Asia-Pacific Microwave Conference (Seoul, South Korea, 2013)

19. G.L. Huang, S.G. Zhou, T.H. Chio, H.T. Hui, T.S. Yeo, IEEE Transactions on Antennas and Propagation 63(1), 419 (2015)

20. Grasp 9.8. URL http://www.ticra.com

21. Ansys HFSS 13. URL http://www.ansoft.com

22. M. Pastorino, Microwave imaging (John Wiley \& Sons, 2010)

23. L.B. Lucy, Astronomical Journal 79(6), 745 (1974)

24. W.H. Richardson, Journal of the Optical Society of America 62(1), 55 (1972)

25. X. Zheng, J.Y. Yang, L.C. Li, Acta Autom. Sin. 35, 28 (2009) 